\title{
Editorial
}

\section{Current Knowledge on Growth Hormone and Insulin-Like Growth Factors and their Role in the Central Nervous System}

\author{
Fred Nyberg ${ }^{*} \S$
}

\begin{abstract}
Department of Pharmaceutical Biosciences, Division of Biological Research on Drug Dependence, Uppsala University, P.O. Box 591, SE-751 24 UPPSALA, Sweden
\end{abstract}

The past two decades have seen a significantly increasing interest for effects that growth hormone (GH) may exert on the central nervous system (CNS). Thus, an increasing number of studies indicating that the hormone may display an important function in many behavioral aspects related to the CNS have emerged from various clinics and basic science laboratories.

GH mediates most of its effects by releasing insulin-like growth factor-1 (IGF-1) through stimulation of GH receptors (GHR), in particular in the liver. Both GH and IGF-1 are shown to cross the blood-brain barrier (BBB) and affect specific targets for these hormones in the brain. The discovery of GH and IGF-1 receptors in the CNS and their distribution in brain areas associated with the functional anatomy of behavioral effects induced by activation of the somatotrophic axis (GH/IGF-1 axis) has brought up new aspects on the mechanisms underlying beneficial effects seen for the two hormones.

In individuals with deficient $\mathrm{GH}$ production, e.g. due to disturbed function of the GH/IGF-1 axis, a reduction of several dimensions related to quality of life (QOL) has been observed. Impaired activity in this axis may results from various types of pathology but also from normal aging processes. In patients with a pathological decline in circulating $\mathrm{GH}$, however, exogenous compensation of the hormone has been seen to counteract these QOL-disabilities.

Indeed, GH replacement therapy has in many clinical studies been evidenced to improve the psychological capabilities in adult GH deficient (GHD) patients. Profound effects of the hormone on certain functions, including memory and learning, mental alertness, motor skills, motivation and working capacity have been reported. Also in GHD children the hormone replacement has been shown to produce significant improvement in many behavioral problems seen in these individuals. In children with primary IGF-1 deficiency, treatment with recombinant IGF-1 has

*Address correspondence to this author at the Department of Pharmaceutical Biosciences, Division of Biological Research on Drug Dependence, Uppsala University, P.O. Box 591, SE-751 24 UPPSALA, Sweden;

Tel: +46-18-471-4166; E-mail: fred.nyberg@farmbio.uu.se

${ }^{\S}$ Guest Editor. been seen to increase the height. According to data from recent studies the growth factor was well tolerated and induced dose-dependent annualized height velocities in children. Also, IGF-1 is shown to be implicated in prenatal and postnatal phases of brain development, including neurogenesis, neuronal differentiation, synaptogenesis, and is shown to restore cortical plasticity in adult life. Therefore, studies on effects of IGF-1 on the brain function are of potential interest.

In addition, effects of IGF-1 on brain function have also been applied in order to explore the mechanism underlying the effects of GH on brain function. The aim with this hot topic issue is to compile and illuminate recent knowledge in this area. This includes clinical observations of patients with reduced ability to produce the hormone but also outcomes of GH and IGF-1 replacement therapy in GHD-patients, neuroprotective effects involving the GH/IGF-1 axis as well as animal models exploring the mechanisms at the neurochemical level by which the hormone induces its effects.

The first chapters of this issue are dealing with some fundamental concepts regarding biosynthesis and chemical properties of GH and IGF-1. They also focus on mechanisms underlying the regulation of secretion of these hormones to give readers a compendious view and background of events taking place in the body before the hormones interact with their receptors. The second chapter also describes basic mechanisms regarding the interactions between $\mathrm{GH}$ and IGF-1 with receptors on their target cells. It pictures the receptor structure and provides detailed information about mechanisms involved in signal transduction leading to the final GH or IGF-1 responses. Subsequent to these two introductory chapters the text addresses aspects on the CNS starting with brief description of GH targets in the brain.

Chapter 3 includes text on the characteristics of GH receptors in the brain. It provides information about the structure and distribution of GHR in the CNS and also discusses functional aspects with respect to their location in various brain regions. Furthermore, views on the ability of the hormones to penetrate the blood-brain barrier are brought up. 
In two subsequent chapters experimental animal models to study the role of GHR and the GH/IGF-1 axis in the CNS are discussed. The use of transgenic models has allowed studies on the function of $\mathrm{GH}$ in brain growth and motorneuron development. Transgenic mice models for specific studies o aging and impairments in cognitive functions are also described.

The remaining chapters are directed to clinical aspects of the GH/IGF- 1 axis in the brain with emphasis on current and future possible therapeutic strategies. The role of $\mathrm{GH}$ and IGF-1 in aging and dementia in human subjects, in psychiatric disorders and in Alzheimers disease are highlighted in this part of the hot topic volume. GH replacement therapy to improve life quality, memory disabilities, as well as motor skills in individuals with Down's syndrome is also discussed.

Although this issue of The Open Journal of Endocrinology is confined to twelve chapters it is interesting to see that it covers the most important aspects of the actual topic. It is worthwhile that it has been possible get together such competent contributors of good worldwide representation, all of them well established in the actual field of research. Although still some unexplored, the role of the GH/IGF-1 axis in the brain represents a challenge for basic science and clinical medicine because it seems to be involved in the regulation of brain growth, development, and metabolism but also in many aspects of brain pathology. Therefore, it is believed that with the current advances in research on GH and IGF-1 this volume will represent a timely issue that contributes an important topic of interest to many scientists, from students and medical practitioners to basic and clinical researchers.

At last, I am grateful to all my colleagues, who have spent time to prepare their excellent contributions to this hot topic issue. As editor, I would like to point out that most of these chapters with a few exceptions were submitted within a time period of about 12 months and they should therefore contribute very recent knowledge in the actual field. 